\title{
Photoreceptor disruption in central serous chorioretinopathy treated by half-dose photodynamic therapy
}

This article was published in the following Dove Press journal:

Clinical Ophthalmology

9 January 2013

Number of times this article has been viewed

\section{Mansing Ratanasukon \\ Koblarp Thongthong \\ Patama Bhurayanontachai \\ Pichai Jirarattanasopa}

Department of Ophthalmology, Faculty of Medicine, Prince of Songkla University, Hat yai, Songkhla Province, Thailand
Correspondence: Mansing Ratanasukon Department of Ophthalmology, Faculty of Medicine, Prince of Songkla University, Hat Yai, Songkhla Province 90I I0, Thailand

Tel +667445 I38 I

Fax +6674429619

Email mratanasukon@yahoo.com
Background: To evaluate photoreceptor disruption in patients with central serous chorioretinopathy (CSC) treated by half-dose photodynamic therapy (PDT).

Methods: A total of 29 patients with symptomatic CSC were recruited and underwent half-dose verteporfin PDT covering the leakage sites as observed via fundus fluorescein angiography. The primary outcome was the percentage of patients with the presence of photoreceptor disruption, and the secondary outcome was the correlation between photoreceptor disruption and visual results at the 1-year follow-up.

Results: Photoreceptor disruption was identified in 13 eyes (44.8\%) 12 months after treatment. Twenty-seven patients experienced best-corrected visual acuity (BCVA) improvement after PDT, while two patients showed stable BCVA. The mean BCVA in patients with photoreceptor disruption at the baseline and every follow-up visit was significantly lower than that of patients without photoreceptor disruption. However, there was no correlation between the presence or absence of photoreceptor disruption and the improvement of visual acuity because the BCVA gain at the last follow-up visit between the two groups was not significant $(P=0.69)$. No potential ocular complication was encountered in the study.

Conclusion: Photoreceptor disruption was found in about $45 \%$ of CSC patients treated by PDT, which ultimately resulted in poor visual outcomes. However, a half-dose PDT might not affect or modify the photoreceptor function because it gave the same pattern of visual recovery in patients with and without photoreceptor cell loss.

Keywords: photoreceptor disruption, central serous chorioretinopathy, photodynamic therapy

\section{Introduction}

Central serous chorioretinopathy (CSC) is an idiopathic condition characterized by the development of a well circumscribed, serous detachment of the neurosensory retina in the macula. This condition is common in healthy young adults between the ages of 20 and 50 years, especially in men with a type A personality, ${ }^{1}$ those under stress, or patients with elevated levels of corticosteroids from either corticosteroid administration or Cushing syndrome. ${ }^{2,3}$ Most patients are asymptomatic unless the central macula is affected. The patients may experience visual disturbances including micropsia, metamorphopsia, central scotoma, reduced visual acuity, loss of contrast sensitivity, and visual loss. ${ }^{4}$ The natural course of acute CSC is good in most cases $(80 \%-90 \%)$ as it usually resolves spontaneously in 3-4 months. ${ }^{5,6}$ However, despite recovery of visual acuity, some patients may experience residual visual symptoms with a loss in contrast sensitivity and, in approximately $30 \%-50 \%$ of patients, recurrence may also take place within the first year. ${ }^{7}$ 
$\mathrm{CSC}$ is postulated to occur as a result of a choriocapillaris leakage through the retinal pigment epithelium (RPE). ${ }^{8}$ The fundus fluorescein angiography (FFA) of acute CSC typically shows focal leaks at the level of the RPE in two main patterns: "inkblot" or "smokestack." The use of indocyanine green angiography in CSC patients has demonstrated that CSC primarily affects the choroidal circulation, resulting in multifocal areas of choroidal vascular hyperpermeability., ${ }^{8,12}$ Moreover, optical coherence tomography (OCT) is an excellent, noninvasive method for diagnosing and following the resolution of the subretinal fluid in CSC. High-resolution OCT can detect subtle fluid accumulation beneath the sensory retina, and not surprisingly, a thickening of the choroid. ${ }^{13}$

The traditional management of acute CSC is usually observation. The laser photocoagulation of a fluoresceindetectable pigment epithelial leak can accelerate the resolution of the associated neurosensory detachment. ${ }^{5,6}$ Although laser may shorten the duration of symptoms, it does not have any impact on the final vision or the recurrent rate of CSC because it targets only the RPE leak without specifically treating the underlying primary choroidal congestion and hyperpermeability. ${ }^{14}$ When the leakage site is too close to the center of the fovea, photodynamic therapy (PDT) with verteporfin is another recent option for treating such CSC. Many studies have demonstrated beneficial visual outcomes in most CSC patients treated by PDT. ${ }^{15-18}$

The mechanism of PDT for treating CSC involves shortterm choriocapillaris hypoperfusion and long-term choroidal vascular remodeling, leading to a reduction in choroidal congestion, vascular hyperpermeability, and leakage. ${ }^{16,19,20}$ The potential complications such as RPE atrophy, choroidal ischemia, and secondary CNV can occur in the application of conventional PDT in CSC..$^{16,18,21}$ By reducing the dose of verteporfin, and altering the timing of infusion and laser application, many studies have demonstrated that the potential retinal damage caused by PDT can be minimized while resulting in good treatment efficacy of chronic CSC. ${ }^{22,23}$ Nevertheless, during the half-dose PDT treatment, damages involving surrounding retinal structures such as RPE or photoreceptor cells might still occur. In this study, photoreceptor damage was measured by detecting photoreceptor disruption via spectral-domain OCT (SD-OCT) in patients with CSC treated by half-dose PDT, and also assessed the correlation between photoreceptor disruption and visual results.

\section{Methods}

This study was a retrospective descriptive study conducted in the Department of Ophthalmology, Songklanagarind Hospital,
Prince of Songkla University, Thailand between March 2006 and February 2011. Patients with symptomatic acute CSC, undergoing PDT using a half-dose of verteporfin (Visudyne; Novartis AG, Bülach, Switzerland) were studied. The patients underwent fundus examinations and had their best-corrected visual acuity (BCVA) measured using the logarithm of the minimum angle of resolution (logMAR). CSC was diagnosed when there was focal retinal detachment with or without pigment epithelial detachment in the macular area and focal point leakage on FFA. The inclusion criteria included: (1) age more than 20 years, (2) typical CSC characteristics confirmed by FFA and OCT, (3) follow-up fundus examinations and OCT tests more than 12 months after the half-dose PDT, (4) single half-dose PDT treatment with no other accompanying treatment, and (5) no recurrent attack after half-dose PDT treatment within 1 year of follow-up. The exclusion criteria included: (1) complicated CSC presentation such as choroidal neovascular membrane, multiple retinal pigment epithelial detachment, large pigment epithelial detachment more than one disc diameter, and diffuse pigment epitheliopathy before the treatment; (2) treatment via focal laser or intravitreal injection before and after the half-dose PDT; and (3) patients with fluorescein and verteporfin allergy.

The FFA was performed on all patients before the halfdose PDT using Spectralis Heidelberg retinal angiography (HRA-2, Heidelberg Engineering, Dossenheim, Germany). The SD-OCT was also performed using a Cirrus-OCT (Carl Zeiss Meditec, Inc, Dublin, CA) before and at 1, 3, 6, and 12 months after PDT. From OCT, the macular thickness analysis program of $6 \times 6 \mathrm{~mm}^{2}$ and 9-subfields analysis was used to identify central macular thickness, subretinal fluid, and any abnormality area.

A reduced dose of verteporfin $\left(3 \mathrm{mg} / \mathrm{m}^{2}\right)^{22,23}$ was infused over 8 minutes, followed by $689-\mathrm{nm}$ laser delivery at 10 minutes after the commencement of infusion to target the area of leakage points. A total light energy of $50 \mathrm{~J} / \mathrm{cm}^{2}$ over 83 seconds was delivered to the area of angiographic leakage sites shown in FFA.

\section{Evaluation of photoreceptor disruption}

At baseline, OCT was performed before PDT (during the active stage or detached retina), but it could not identify the photoreceptor line in all cases because of low signal and/or disorganized retinal structures. Therefore, the presence of photoreceptor disruption was only diagnosed 12 months after the half-dose PDT. The diagnosis of the disruption was based on either of the two OCT-characteristic patterns: (1) discontinuity of the inner segment/outer segment (IS/OS) 
line from SD-OCT (Figure 1) and (2) the total absence of the IS/OS line (Figure 2). These SD-OCT findings were evaluated by three authors (MR, PB, and PJ); two out of the three consensus opinions were necessary to make the decision on whether the photoreceptor disruption was present.

The data obtained included age at first visit, gender, laterality of the eyes, BCVA, duration of symptoms, and the presence of photoreceptor disruption at 12 months follow-up. The percentage of patients with the presence of photoreceptor disruption was considered as a primary outcome, and the secondary outcome comprised the correlation between photoreceptor disruption and visual results.

\section{Statistical analysis}

The descriptive analysis was used for reporting and explaining the presence of photoreceptor disruption. The correlation of visual results and the presence of photoreceptor disruption were analyzed using the independent samples $t$-test.

\section{Results}

A total of 29 eyes from 29 patients (27 males and 2 females) were reviewed. The mean age was 46.2 years (range 33-62 years), and the mean duration of symptoms before treatment was 192 days (range 13-1097 days) (Tables 1 and 2). The mean durations of the disease in patients with and without photoreceptor disruption were 205 and 182 days $(P=0.8)$ (Table 2). The mean logMAR BCVA scores of all the patients at baseline and 12 months were 0.34 (standard deviation $[\mathrm{SD}] \pm 0.30$ ) and 0.14 (SD \pm 0.27 ), respectively. Twenty-seven patients experienced a BCVA improvement after PDT, whereas two patients showed stable BCVA. Regarding the primary outcome, photoreceptor disruption at the fovea was observed in 13 eyes (44.8\%) 12 months after treatment.

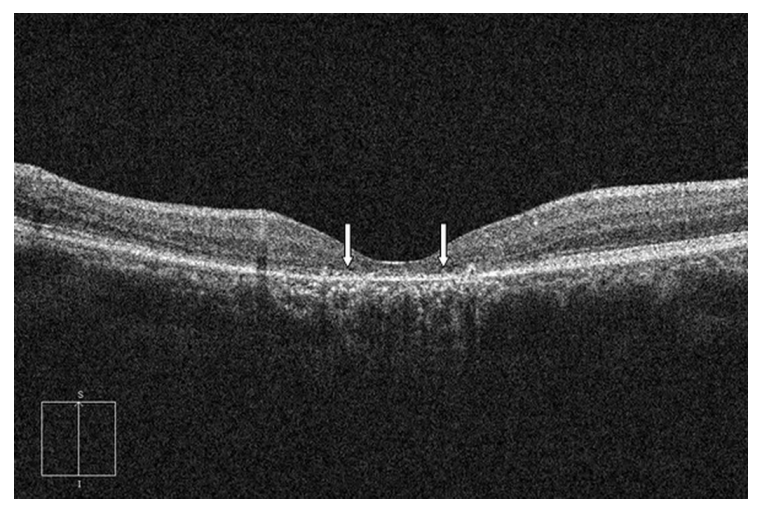

Figure I The optical coherence tomography of a patient with photoreceptor disruption demonstrated the discontinuity of the inner segment/outer segment line (arrows).

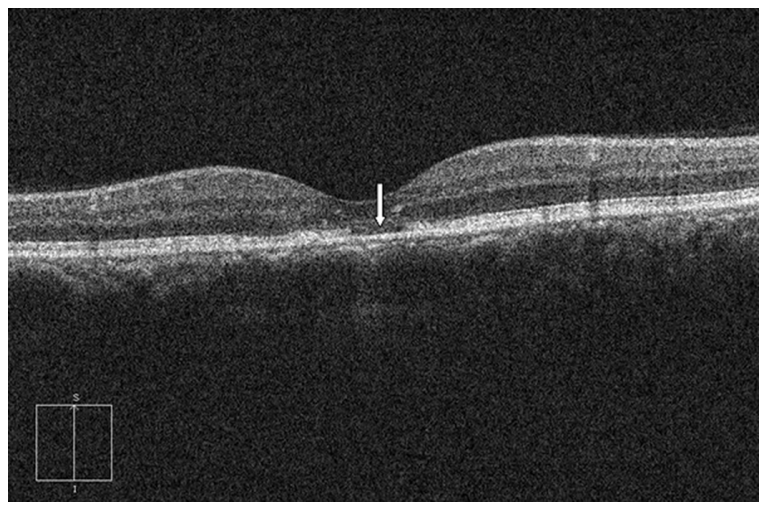

Figure 2 The optical coherence tomography of a patient with photoreceptor disruption demonstrated total absence of inner segment/outer segment line (arrow).

Photodynamic therapy with verteporfin resulted in a rapid resolution of subretinal fluid in all patients as demonstrated by OCT. The difference of central macular thickness between the patients with or without photoreceptor disruption was not significant (Table 2). However, the mean BCVA in patients with the presence of photoreceptor disruption at baseline and 12 months after treatment was lower than that of patients with intact photoreceptor cells (Table 2 and Figure 3). The difference in BCVA at baseline and every visit (1, 3, 6, and 12 months) between the two groups was statistically significant (Table 2 and Figure 3 ) but the visual acuity gain at the last follow-up visit between the two groups was not significant $(0.19$ and $0.22, P=0.69)$ (Table 2). At 12 months post treatment, the mean BCVA of the patients without photoreceptor disruption further improved to 0.01 ( $\mathrm{SD} \pm 0.03$ ), whereas that in the patients with photoreceptor disruption was only $0.31(\mathrm{SD} \pm 0.34)$. All patients without photoreceptor disruption resulted in visual acuity improvement, whereas only eleven patients (84.6\%) with photoreceptor disruption showed visual acuity improvement. In respect to the secondary outcome, there was no correlation

Table I Demographic data

\begin{tabular}{ll}
\hline & Data \\
\hline $\begin{array}{l}\text { Mean age (years } \pm \text { SD) } \\
\text { Male }\end{array}$ & $46.24 \pm 7.16$ \\
$\quad$ Female & $27(93.1)$ \\
Final BCVA (patients, \%) & $2(6.9)$ \\
$\quad$ Improved BCVA & $27(93.1)$ \\
$\quad$ Stable BCVA & $2(6.9)$ \\
$\begin{array}{l}\text { Presence of photoreceptor disruption at I2 months } \\
\text { (patients, \%) }\end{array}$ \\
$\quad \begin{array}{l}\text { Present } \\
\quad \text { Absent }\end{array}$ \\
\hline Abbreviations: SD, standard deviation; BCVA, best-corrected visual acuity.
\end{tabular}

Abbreviations: SD, standard deviation; BCVA, best-corrected visual acuity. 
Table 2 Study results

\begin{tabular}{|c|c|c|c|}
\hline \multirow[t]{2}{*}{ Variable } & \multicolumn{2}{|c|}{ Photoreceptor disruption } & \multirow[t]{2}{*}{$P$-value } \\
\hline & Present & Absent & \\
\hline Mean duration of symptoms (days \pm SD) & $205.00 \pm 197.3$ & $|82.63 \pm 260.6|$ & 0.800 \\
\hline \multicolumn{4}{|l|}{ Mean BCVA (logMAR $\pm S D)$} \\
\hline At baseline & $0.52 \pm 0.35$ & $0.20 \pm 0.13$ & 0.006 \\
\hline At I month & $0.42 \pm 0.37$ & $0.10 \pm 0.09$ & 0.009 \\
\hline At 3 months & $0.33 \pm 0.35$ & $0.04 \pm 0.05$ & 0.010 \\
\hline At 6 months & $0.34 \pm 0.38$ & $0.03 \pm 0.05$ & 0.029 \\
\hline At 12 months & $0.31 \pm 0.34$ & $0.01 \pm 0.03$ & 0.008 \\
\hline Mean BCVA improvement $(\log M A R \pm S D)$ & $0.22 \pm 0.24$ & $0.19 \pm 0.13$ & 0.694 \\
\hline \multicolumn{4}{|l|}{ Mean central macular thickness from OCT (micron \pm SD) } \\
\hline At baseline & $333.50 \pm 74.25$ & $439.00 \pm 106.99$ & 0.267 \\
\hline At I month & $196.00 \pm 30.61$ & $228.00 \pm 19.80$ & 0.400 \\
\hline At 3 months & $174.25 \pm 42.72$ & $225.00 \pm 18.22$ & 0.032 \\
\hline At 6 months & $187.00 \pm 49.19$ & $224.70 \pm 22.68$ & 0.118 \\
\hline At 12 months & $192.25 \pm 62.02$ & $233.7 \mid \pm I 5.27$ & 0.412 \\
\hline Mean central macular thickness improvement from OCT (micron \pm SD) & $135.00 \pm 38.18$ & $160.00 \pm 118.79$ & 1.000 \\
\hline
\end{tabular}

Abbreviations: SD, standard deviation, BCVA, best-corrected visual acuity, logMAR, logarithm of minimum angle of resolution; OCT, optical coherence tomography.

between the presence or absence of photoreceptor disruption and visual acuity improvement.

At 12 months post treatment, no potential ocular complications, such as secondary choroidal neovascularization and RPE tear, were found in any of the patients. Finally, there was no recurrence of CSC during the 1-year follow-up period.

\section{Discussion}

Various medical treatments have been used to treat CSC without success. Laser treatment is a standard modality in CSC management, which involves the debridement of RPE and permitting the ingrowth of surrounding healthy
RPE and an induced rapid resolution of CSC. However, the leaks are often numerous and might be too close to the fovea to allow safe laser treatment. It may, therefore, lead to complications such as accidental photocoagulation of macula, foveal distortion, scotomas, significant loss of contrast sensitivity, and choroidal neovascularization. More recently, a standard dose of verteporfin in photodynamic therapy has proven effective for the treatment of longstanding CSC and CSC with leakage at the macula, as it has resulted in subretinal fluid disappearance and complete resolution of all symptoms. However, potential adverse events like choroidal ischemia, retinal pigment epithelium atrophy

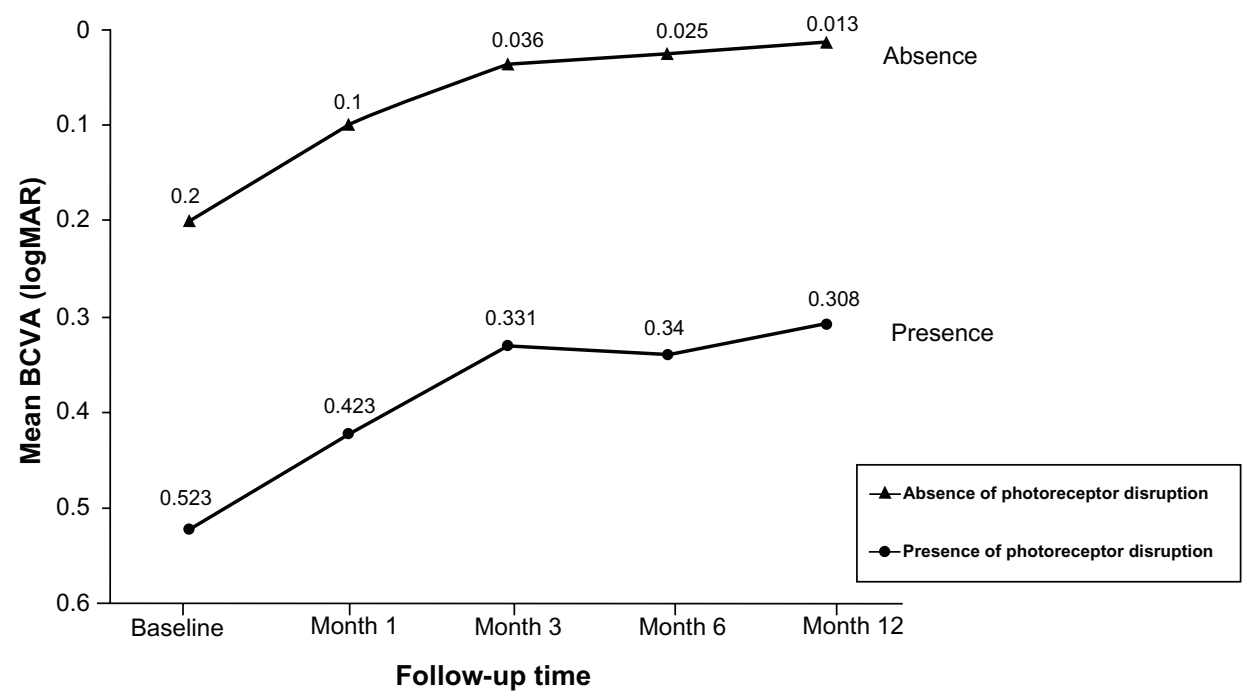

Figure 3 The mean visual acuity changes in central serous chorioretinopathy patients with and without photoreceptor disruption treated by half-dose photodynamic therapy. Abbreviations: BCVA, best-corrected visual acuity, logMAR, logarithm of minimum angle of resolution. 
and secondary choroidal neovascularization may develop after such treatment. To enhance the efficacy of PDT in treating CSC, while minimizing its side effects, the dosage of verteporfin was reduced and the interval between infusion and laser application shortened. After the treatments, 27 patients $(93.1 \%)$ demonstrated BCVA improvement and mean $\log$ MAR BCVA gain in all patients was about 0.2 (Tables 1 and 2). These results were comparable with previous studies..$^{22,23}$

The present study found photoreceptor disruption at the fovea in $44.8 \%$ of CSC cases treated by half-dose PDT at 12 months. The disruption might be due to PDT, the resolution process, or even the disease chronicity itself. However, it was very difficult to identify the disruption caused by OCT before the treatment or in the presence of active subretinal fluid because either the retinal signal was low or the retinal structures were disorganized and led to difficulties related to IS/OS line identification using OCT. Ojima et $\mathrm{al}^{24}$ have reported the same difficulties involving active CSC cases. They found that the photoreceptor layer reappeared after reattachment of the retina even where no back reflection from the IS/OS line was seen during the active stage. This finding suggests that it is almost impossible to accurately identify the photoreceptor status during an active or detached CSC retina. In the present study, the line was able to be identified only after the treatment (after resolution of fluid) and a disrupted line was found in $44.8 \%$ of the eyes, as described. However, regardless of the duration of symptoms, the baseline visual acuity of patients with a IS/OS disrupted line was significantly lower than that of patients with an intact line $(0.52$ and $0.20, P=0.006)$ (Table 2 and Figure 3). These findings suggested that the photoreceptor cell loss might occur before any treatment, not related to PDT, and lead to lower visual outcomes in every visit when compared with the other group.

Concerning the visual recovery and final visual outcomes, the visual acuity at 1 year of patients with photoreceptor disruption was significantly lower than that of the intact group (0.31 and 0.01, $P=0.008$ ) (Table 2 and Figure 3). However, there was no correlation between the presence or absence of photoreceptor disruption and the improvement of visual acuity because the BCVA gain at the last follow-up between the two groups was not significant ( 0.22 and 0.19 , $P=0.69$ ). This means that the pattern of visual recovery in both groups demonstrated an improvement in the same direction (Figure 3). Moreover, these findings also suggest that either PDT did not seem to affect photoreceptor cell function, or that photoreceptor cell loss might happen before
PDT and the treatment did not affect their integrity. Thus, the lower final visual outcomes may be attributed to pre-PDT lower photoreceptor function or cell loss.

The limitations of this study involve its small sample size and the lack of comparison between patients with and without PDT. As described above, the unreliability related to IS/OS line identification during the active stage is another limitation. Furthermore, the assumption of the effects of PDT on photoreceptor cells only depended on the pattern of visual recovery and the final visual outcomes at 1 year.

\section{Conclusion}

In conclusion, photoreceptor disruption was observed in $44.8 \%$ of CSC patients treated by PDT, which ultimately resulted in poor visual outcomes. Nevertheless, the half-dose PDT treatment might not affect or modify photoreceptor function, and it yields the same pattern of visual recovery in patients with and without photoreceptor cell loss.

\section{Ethics approval}

The article was approved by the Ethics Committee, Faculty of Medicine, Prince of Songkla University on March 6, 2012 (EC 55-161-02-1-3).

\section{Acknowledgment}

The authors would like to thank Mansing Ratanasukon for substantial contribution to conception and design, analysis and interpretation of data, drafting the article, and final approval of the published version. The authors would also like to thank Koblarp Thongthong, Patama Bhurayanontachai, and Pichai Jirarattanasopa for acquisition of data.

\section{Disclosure}

The authors report no conflicts of interest in this work.

\section{References}

1. Yannuzzi LA. Type-A behavior and central serous chorioretinopathy. Retina. 1987;7:111-131.

2. Carvalho-Recchia CA, Yannuzzi LA, Negrão S, et al. Corticosteroids and central serous chorioretinopathy. Ophthalmology. 2002;109: 1834-1837.

3. Fernandez CF, Mendoza AJ, Arevola JF. Central serous chorioretinopathy associated with topical dermal corticosteroids. Retina. 2004;24: 471-474.

4. Gass JD. Pathogenesis of disciform detachment of neuroepithelium. Am J Ophthalmol. 1967;63:1-139.

5. Gass JDM. Photocoagulation treatment of idiopathic central serous chorioretinopathy. Trans Am Acad Ophthalmol Otolaryngol. 1977;83: 456-467.

6. Yannuzzi LA. Type A behavior and central serous chorioretinopathy. Trans Am Ophthalmol Soc. 1986;84:799-845.

7. Folk JC, Thompson HS, Han DP, et al. Visual function abnormalities in central serous retinopathy. Arch Ophthalmol. 1984;102:1299-1302. 
8. Spaide RF, Hall L, Haas A, et al. Indocyanine green videoangiography of older patients with central serous chorioretinopathy. Retina. 1996;16: 203-213.

9. Maumenee AE. Fluorescein angiography in the diagnosis and treatment of lesions of the ocular fundus. Trans Ophthalmol Soc U K. 1968;88: 529-556.

10. Prunte C, Flammer J. Choroidal capillary and venous congestion in central serous chorioretinopathy. Am J Ophthalmol. 1996;121:26-34.

11. Guyer DR, Yannuzzi LA, Slakter JS, et al. Digital indocyanine green videoangiography of central serous chorioretinopathy. Arch Ophthalmol. 1994;112:1057-1062.

12. Piccolino FC, Borgia L. Central serous chorioretinopathy and indocyanine green angiography. Retina. 1994;14:231-242.

13. Spaide RF, Koizumi H, Pozzoni MC. Enhanced depth imaging spectral-domain optical coherence tomography. Am J Ophthalmol. 2008;146:496-500.

14. Robertson DM, Ilstrup D. Direct, indirect, and sham laser photocoagulation in the management of central serous chorioretinopathy. Am J Ophthalmol. 1983;95:457-466.

15. Ober MD, Yannuzzi LA, Do DV, et al. Photodynamic therapy for focal retinal pigment epithelial leaks secondary to central serous chorioretinopathy. Ophthalmology. 2005;12:2088-2094.

16. Chan WM, Lam DS, Lai TY, et al. Choroidal vascular remodelling in central serous chorioretinopathy after indocyanine green guided photodynamic therapy with verteporfin: a novel treatment at the primary disease level. Br J Ophthalmol. 2003;87:1453-1458.
17. Yannuzzi LA, Slakter JS, Gross NE, et al. Indocyanine green angiography-guided photodynamic therapy for treatment of chronic central serous chorioretinopathy: a pilot study. Retina. 2003;23:288-298.

18. Cardillo Piccolino F, Eandi CM, Ventre L, et al. Photodynamic therapy for chronic central serous chorioretinopathy. Retina. 2003;23: $752-763$.

19. Schlotzer-Schrehardt U, Viestenz A, Naumann GO, et al. Dose-related structural effects of photodynamic therapy on choroidal and retinal structures of human eyes. Graefes Arch Clin Exp Ophthalmol. 2002;240: 748-757.

20. Schmidt-Erfurth U, Laqua H, Schlotzer-Schrehard U, et al. Histopathological changes following photodynamic therapy in human eyes. Arch Ophthalmol. 2002;120:835-844.

21. Colucciello M. Choroidal neovascularization complicating photodynamic therapy for central serous retinopathy. Retina. 2006;26:239-242.

22. Chan WM, Lai TY, Lai RY, et al. Safety enhanced photodynamic therapy for chronic central serous chorioretinopathy: one-year results of a prospective study. Retina. 2008;28:85-93.

23. Chan WM, Lai TY, Lai RY, et al. Half-dose verteporfin photodynamic therapy for acute central serous chorioretinopathy: one-year results of a randomized controlled trial. Ophthalmology. 2008;115:1756-1765.

24. Ojima Y, Hangai M, Sasahara M, et al. Three-dimensional imaging of the foveal photoreceptor layer in central serous chorioretinopathy using high-speed optical coherence tomography. Ophthalmology. 2007;114: 2197-2207.
Clinical Ophthalmology

\section{Publish your work in this journal}

Clinical Ophthalmology is an international, peer-reviewed journal covering all subspecialties within ophthalmology. Key topics include: Optometry; Visual science; Pharmacology and drug therapy in eye diseases; Basic Sciences; Primary and Secondary eye care; Patient Safety and Quality of Care Improvements. This journal is indexed on

\section{Dovepress}

PubMed Central and CAS, and is the official journal of The Society of Clinical Ophthalmology (SCO). The manuscript management system is completely online and includes a very quick and fair peer-review system, which is all easy to use. Visit http://www.dovepress.com/ testimonials.php to read real quotes from published authors. 